\title{
Products of locally cyclic groups
}

\author{
Bernhard Amberg(D) AND YAROSLAV SysAK
}

\begin{abstract}
We consider groups of the form $G=A B$ with two locally cyclic subgroups $A$ and $B$. The structure of these groups is determined in the cases when $A$ and $B$ are both periodic or when one of them is periodic and the other is not. Together with a previous study of the case where $A$ and $B$ are torsion-free, this gives a complete classification of all groups that are the product of two locally cyclic subgroups. As an application, it is shown that the Prüfer rank of a periodic product of two locally cyclic subgroups does not exceed 3, and this bound is sharp. It is also proved that a product of a finite number of pairwise permutable periodic locally cyclic subgroups is a locally supersoluble group. This generalizes a well-known theorem of B. Huppert for finite groups.
\end{abstract}

Mathematics Subject Classification. 20D40.

Keywords. Products of groups, Soluble group, Locally supersoluble group, Locally cyclic group, Prüfer rank.

1. Introduction. Let the group $G=A B$ be the product of two subgroups $A$ and $B$, i.e. $G=\{a b \mid a \in A, b \in B\}$. It was proved by N. Itô that the group $G$ is metabelian if the subgroups $A$ and $B$ are abelian (see [1, Theorem 2.1.1]). This result laid the foundation for a systematic study of groups of the form $G=A B$ with various conditions on the subgroups $A$ and $B$. In particular, it follows directly from Itô's result that every periodic group $G=A B$ with abelian subgroups $A$ and $B$ is locally finite. It is also well-known that the group $G=A B$ with cyclic subgroups $A$ and $B$ is supersoluble and abelian-byfinite ([1, Lemma 7.4.6]). Furthermore, a detailed description of the structure of the group $G=A B$ with torsion-free locally cyclic subgroups $A$ and $B$ was obtained by the second author in [9].

The aim of this paper is to describe the structure of groups which are products of two locally cyclic subgroups in the periodic and the mixed case. Altogether this gives a complete answer to [1, Question 15]. 
Theorem 1.1. Let the periodic group $G=A B$ be the product of two locally cyclic subgroups $A$ and $B$. Then $G$ contains uniquely determined locally cyclic normal subgroups $S$ and $T$ and a locally nilpotent subgroup $H=A^{*} B^{*}$ with $A^{*} \leq A$ and $B^{*} \leq B$ such that

$$
G=(S \times T) \rtimes H=\left(S \times A^{*}\right)\left(T \times B^{*}\right)
$$

where $\pi(S) \cap \pi\left(A^{*}\right)=\pi(T) \cap \pi\left(B^{*}\right)=\emptyset, S=\left[S, B^{*}\right]$, and $T=\left[T, A^{*}\right]$.

We recall that a group $G$ has finite Prüfer rank $r=r(G)$ (special rank in the sense of Mal'cev in Russian terminology) if each finitely generated subgroup of $G$ can be generated by $r$ elements and $r$ is the least positive integer with this property. Clearly a group is of rank 1 if and only if it is locally cyclic. It is also known that every finite $p$-group of the form $G=A B$ with cyclic subgroups $A$ and $B$ has rank at most 2 for $p$ odd [5, Satz 8] and at most 3 for $p=2[6$, Theorem 5.1]. The following consequence of Theorem 1.1 gives an exact upper bound for the Prüfer rank of the product $G=A B$ of two periodic locally cyclic subgroups $A$ and $B$.

Corollary 1.2. If $G=A B$ is a periodic group with locally cyclic subgroups $A$ and $B$, then the Prüfer rank of $G$ does not exceed 3 .

It should be noted that this result is also new for arbitrary finite groups of the form $G=A B$ with cyclic subgroups $A$ and $B$.

Finally, the following theorem extends a well-known result of B. Huppert on the supersolubility of finite groups which are products of pairwise permutable cyclic subgroups (see [5, Satz 34] or [4, Satz VI.10.3]). This gives, in particular, an affirmative answer to [2, Question 1].

Theorem 1.3. Let the group $G=A_{1} A_{2} \cdots A_{n}$ be the product of finitely many pairwise permutable periodic locally cyclic subgroups $A_{1}, \ldots, A_{n}$. Then $G$ is a periodic locally supersoluble group.

2. Preliminaries. In what follows $G=A B$ is a group with locally cyclic subgroups $A$ and $B$.

Lemma 2.1. If $G=A B$ is an infinite p-group, then up to a permutation of the factors $A$ and $B$ the subgroup $A$ is quasicyclic and one of the following statements hold:

(1) $G=A \times B$ with $B$ cyclic or quasicyclic;

(2) $p=2$ and $G=A \rtimes\langle b\rangle$ for some element $b \in G$ with $a^{b}=a^{-1}$ for all $a \in A$;

(3) $p=2$ and $G=A\langle b\rangle$ for some element $b \in G$ with $b^{2^{n}}=1$ for some $n>1, b^{2^{n-1}} \in A$ and $a^{b}=a^{-1}$ for all $a \in A$.

Proof. Clearly without loss of generality we may assume that the subgroup $A$ is infinite and so quasicyclic. Then the subgroup $B$ is either cyclic or quasicyclic. Since in the latter case the group $G$ is abelian by [1, Lemma 7.4.4], the subgroup $A$ is complemented in $G$ and hence statement (1) holds.

Let the group $G$ be non-abelian. Then the subgroup $B$ is cyclic and so $A$ as a quasicyclic $p$-subgroup of finite index in $G$ must be normal and non-central in 
$G$. In particular, $B$ induces on $A$ a non-trivial cyclic $p$-group of automorphisms. On the other hand, since quasicyclic $p$-groups have no automorphisms of order $p>2$, it follows that $p=2$. But then $B=\langle b\rangle$ with $b^{2^{n}}=1$ for some $n \geq 1$ and $b$ induces on $A$ an automorphism of order 2 that inverts the elements of $A$. In particular, if $A \cap B=1$, we obtain statement (2). In the second case, $A \cap B=\left\langle b^{2^{n-1}}\right\rangle$ and hence statement (3) holds, as claimed.

Corollary 2.2. If $G=A B$ is a p-group and $C, D$ are subgroups of $A$ and $B$, respectively, then $C D=D C$ and so $C D$ is a subgroup of $G$.

Proof. This is known if $G$ is finite (see [5, Satz 3]), and follows from Lemma 2.1 in the general case.

Lemma 2.3. If the group $G=A B$ is periodic and $H$ is a finite subgroup of $G$, then $H$ is contained in a finite subgroup $E$ of $G$ such that $E=(A \cap E)(B \cap E)$. In particular, the group $G$ is locally supersoluble.

Proof. Since the group $H$ is finite, there exist finite subsets $C$ of $A$ and $D$ of $B$ such that $H$ is contained in the set $C D$. Then the subgroups $A_{0}=\langle C\rangle, B_{0}=$ $\langle D\rangle$, and $\langle C, D\rangle$ are finite, because the group $G$ is locally finite. Furthermore, it follows from [1, Lemma 1.2.3] that the normalizer $N_{G}\left(\left\langle A_{0}, B_{0}\right\rangle\right)$ contains a finite subgroup $E$ such that $\left\langle A_{0}, B_{0}\right\rangle \leq E=(A \cap E)(B \cap E)$. Since the subgroup $E$ is supersoluble by [1, Lemma 7.4.6] and $H \subseteq C D \subseteq\langle C, D\rangle=\left\langle A_{0}, B_{0}\right\rangle$, the lemma is proved.

As a direct consequence of this lemma, we have

Corollary 2.4. If the group $G=A B$ is periodic, then there exists an ascending series of finite subgroups $1=G_{0}<G_{1}<\cdots<G_{n}<\cdots G$ such that $G_{n}=$ $\left(A \cap G_{n}\right)\left(B \cap G_{n}\right)$ for each $n>0$ and $G=\bigcup_{n=1}^{\infty} G_{n}$.

If $G$ is a periodic group and $\pi$ is a set of primes, then a subgroup $H$ of $G$ is called a $\pi$-subgroup provided that all prime divisors of the order of any element of $H$ are contained in $\pi$. By a Sylow $\pi$-subgroup of $G$ we simply mean a maximal $\pi$-subgroup $G_{\pi}$ of $G$ which will be denoted by $G_{p}$ if $\pi=\{p\}$.

Lemma 2.5. Let $G=A B$ be a periodic group and $\pi$ a set of primes. Then the following statements hold.

(1) If $A_{\pi}$ and $B_{\pi}$ are Sylow $\pi$-subgroups of $A$ and $B$, respectively, then $G_{\pi}=$ $A_{\pi} B_{\pi}$ is a Sylow $\pi$-subgroup of $G$ and

$$
N_{G}\left(G_{\pi}\right)=N_{A}\left(G_{\pi}\right) N_{B}\left(G_{\pi}\right) .
$$

(2) If $p, q$ are primes with $p>q$, then a Sylow $p$-subgroup $G_{p}$ is normalized by a Sylow q-subgroup $G_{q}$. In particular, $G_{\{p, q\}}=G_{p} G_{q}$ for any primes $p$ and $q$.

Proof. (1) It follows from [1, Lemma 1.3.2] that in the notation of Corollary 2.4 for each $n \geq 1$ the set $\left(A_{\pi} \cap G_{n}\right)\left(B_{\pi} \cap G_{n}\right)$ is a Hall $\pi$-subgroup of $G_{n}$. Therefore $G_{\pi}=\bigcup_{n=1}^{\infty}\left(A_{\pi} \cap G_{n}\right)\left(B_{\pi} \cap G_{n}\right)$ is a Sylow $\pi$-subgroup of $G$. Since $A_{\pi}=\bigcup_{n=1}^{\infty}\left(A_{\pi} \cap G_{n}\right)$ and $B_{\pi}=\bigcup_{n=1}^{\infty}\left(B_{\pi} \cap G_{n}\right)$, this implies $G_{\pi}=A_{\pi} B_{\pi}$. In addition, applying [1, Lemma 1.2.2], we have $N_{G}\left(G_{\pi}\right)=N_{A}\left(G_{\pi}\right) N_{B}\left(G_{\pi}\right)$. 
(2) If $\pi=\{p, q\}$ for some primes $p>q$, then $A_{\{p, q\}}=A_{p} \times A_{q}, B_{\{p, q\}}=$ $B_{p} \times B_{q}$, and $G_{\{p, q\}}=\left(A_{p} \times A_{q}\right)\left(B_{p} \times B_{q}\right)$. As $G$ and so its subgroup $G_{\{p, q\}}$ is locally supersoluble by Lemma 2.3 , the Sylow $p$-subgroup $G_{p}=A_{p} B_{p}$ is normal in $G_{\{p, q\}}$. Therefore, $G_{\{p, q\}}=\left(A_{p} \times A_{q}\right)\left(B_{p} \times B_{q}\right)=G_{p} A_{q} B_{q}=G_{p} G_{q}$, as claimed.

A Sylow basis of a periodic group $G$ is defined to be a complete set $\mathbf{S}=\left\{G_{p}\right\}$ of Sylow $p$-subgroups of $G$, one for each prime $p$, such that $G_{p} G_{q}=G_{q} G_{p}$ for all pairs $p, q$ of primes, and $G_{\pi}=\left\langle G_{p} \mid p \in \pi\right\rangle$ is a Sylow $\pi$-subgroup of $G$ for each set $\pi$ of primes. As is well-known (see [3, Lemma 2.1]), every countable periodic locally soluble group possesses Sylow bases. The basis normalizer $N_{G}(\mathbf{S})$ of a Sylow basis $\mathbf{S}$ of $G$ is by definition the intersection $N_{G}(\mathbf{S})=\bigcap_{p} N_{G}\left(G_{p}\right)$ of the normalizers $N_{G}\left(G_{p}\right)$ of the Sylow $p$-subgroups $G_{p}$ of $\mathbf{S}$ for all $p$.

Lemma 2.6. Let the group $G=A B$ be periodic and $G_{p}=A_{p} B_{p}$ for each prime p. Then $\mathbf{S}=\left\{G_{p}\right\}$ is a Sylow basis of $G$. Moreover, if $A^{*}=\bigcap_{p} N_{A}\left(G_{p}\right)$ and $B^{*}=\bigcap_{p} N_{B}\left(G_{p}\right)$, then $N_{G}(\mathbf{S})=A^{*} B^{*}$.

Proof. Indeed, by Lemma 2.5, the set $\mathbf{S}=\left\{G_{p}\right\}$ forms a Sylow basis of $G$ and $N_{G}\left(G_{p}\right)=N_{A}\left(G_{p}\right) N_{B}\left(G_{p}\right)$ for every $p$ by [1, Lemma 1.2.2]. Therefore, $N_{G}(\mathbf{S})=\bigcap_{p} N_{G}\left(G_{p}\right)=\bigcap_{p} N_{A}\left(G_{p}\right) N_{B}\left(G_{p}\right)$ and it is easy to check that $\bigcap_{p} N_{A}\left(G_{p}\right) N_{B}\left(G_{p}\right)=\left(\bigcap_{p} N_{A}\left(G_{p}\right)\right)\left(\bigcap_{p} N_{B}\left(G_{p}\right)\right)=A^{*} B^{*}$ (see [1, Lemma 1.1.2]). Therefore $N_{G}(\mathbf{S})=A^{*} B^{*}$.

The following lemma is a direct consequence of a well-known result of $\mathrm{L}$. Kovacs (see [7, Theorem 2]).

Lemma 2.7. Let $G$ be a finite soluble group, $\pi$ a set of primes and $H$ a Hall $\pi$-subgroup of $G$. If for each $p \in \pi$ the Prüfer rank of a Sylow p-subgroup of $G$ does not exceed $r$, then $H$ is a subgroup of rank at most $r+1$.

Proof. Indeed, it is obvious that if $K$ is a subgroup of $H$, then every Sylow subgroup of $K$ is generated by $r$ elements. Therefore, $K$ can be generated by $r+1$ elements by the result of Kovacs cited above. Thus every subgroup of $H$ is generated by $r+1$ elements and so $H$ has rank at most $r+1$.

3. Proof of Theorem 1.1. First of all, it follows from Lemma 2.1 that for each prime $p$ every Sylow $p$-subgroup of $G=A B$ satisfies the minimal condition for subgroups. Therefore, $G$ satisfies the minimal condition for $p$-subgroups for all primes $p$. Since the group $G$ is metabelian by Ito's theorem, the locally nilpotent residual $R$ of $G$ is contained in its derived subgroup $G^{\prime}$ and so it is abelian. It was proved by Hartley [3, Theorem 1] that in this case $G=R \rtimes H$, where $H$ is any basis normalizer of $G$. In particular, by Lemma 2.6, we can take $H=A^{*} B^{*}$.

It is easy to see that the subgroup $H$ is locally nilpotent and contains the center $Z(G)$ of $G$. Furthermore, $G^{\prime}=R \times H^{\prime}$ and so $H^{\prime}$ is a normal subgroup of $G$. Since $R$ is abelian and $N_{G}(H)=N_{R}(H) \times H$, it follows that $N_{R}(H) \leq Z(G) \leq H$. Therefore, $N_{R}(H)=1$ and hence $H=N_{G}(H)$. We now show that the subgroup $H=A^{*} B^{*}$ commutes with both subgroups $A$ and $B$. 
Indeed, put $S=R \cap\left\langle A, B^{*}\right\rangle$ and $T=R \cap\left\langle A^{*}, B\right\rangle$. It is clear that $S$ and $T$ are normal subgroups of $G,\left\langle A, B^{*}\right\rangle=S \rtimes H$, and $\left\langle A^{*}, B\right\rangle=T \rtimes H$. On the other hand, as $G=A B$, we have also $\left\langle A, B^{*}\right\rangle=A B_{1}$ and $\left\langle A^{*}, B\right\rangle=A_{1} B$ for some subgroups $A_{1}$ and $B_{1}$ such that $A^{*} \leq A_{1} \leq A$ and $B^{*} \leq B_{1} \leq B$. From here, we deduce $A B_{1} \cap A_{1} B=A_{1} B_{1}=(S \cap T) \rtimes H$. Moreover, passing to the factor group $G / H^{\prime}$, we may restrict ourselves to the case when the subgroup $H=A^{*} B^{*}$ is abelian. Then the subgroups $A^{*}$ and $B^{*}$ centralize $S$ and $T$, respectively, and so the subgroup $H$ centralizes the intersection $S \cap T$. Since $H=N_{G}(H)$, this implies $S \cap T=1$. Thus $A_{1} B_{1}=H=A^{*} B^{*}$ and hence $\left\langle A, B^{*}\right\rangle=A H=A B^{*}$ and $\left\langle A^{*}, B\right\rangle=B H=A^{*} B$, as asserted.

Further, taking into account the equalities $A B^{*}=S \rtimes H$ and $H=A^{*} B^{*}$, we conclude that the subgroup $A^{*}$ centralizes $S$, because $\left[A^{*}, S\right] \leq H^{\prime} \cap S=1$. Since in this case the normalizer $N_{S}\left(B^{*}\right)$ is contained in $N_{G}(H)=H$, we have $N_{S}\left(B^{*}\right)=1$. Therefore, every element $b \in B^{*}$ induces on $S$ an automorphism leaving only the identity element fixed. But then every element of $S$ can be written in the form $b^{-1} s^{-1} b s$ with $s \in S$ and hence $S=\left[B^{*}, S\right]$. Similarly, using the equality $A^{*} B=T \rtimes H$, we derive $T=\left[A^{*}, T\right]$.

Finally, we put $A_{0}=A \cap B S$ and $B_{0}=A T \cap B$. Clearly from the equalities $G=A B, A B^{*}=S \rtimes H$, and $A^{*} B=T \rtimes H$, it follows that $G=S \rtimes A^{*} B=$ $T \rtimes A B^{*}, A=A^{*} \times A_{0}$, and $B=B^{*} \times B_{0}$. Therefore, $S \rtimes B=A_{0} B$ and $T \rtimes A=$ $A B_{0}$. Furthermore, if $S_{p}$ is a Sylow $p$-subgroup of $S$, then $S_{p} \rtimes B=\left(A_{0} \cap S_{p} B\right) B$ and if $S_{p} \neq 1$, then $A_{0} \cap S_{p} B \neq 1$. Since the subgroup $A_{0}$ is locally cyclic, this implies that $S$ is locally cyclic and $\pi(S)$ is contained in $\pi\left(A_{0}\right)$. Moreover, as $A^{*}$ and $A_{0}$ are subgroups of the locally cyclic subgroup $A$, it also follows that $\pi\left(A^{*}\right) \cap \pi\left(A_{0}\right)=\emptyset$. Similarly, using the equality $T \rtimes A=A B_{0}$, we obtain $\pi(T)=\pi\left(B_{0}\right)$ and $\pi\left(B^{*}\right) \cap \pi\left(B_{0}\right)=\emptyset$.

Proof of Corollary 1.2. By Corollary 2.4, we may restrict ourselves to the case in which the group $G=A B$ is finite. By Theorem 1.1, $G$ then contains cyclic normal subgroups $S$ and $T$ and a nilpotent subgroup $H=A^{*} B^{*}$ with $A^{*} \leq A$ and $B^{*} \leq B$ such that

$$
G=(S \times T) \rtimes H=\left(S \times A^{*}\right)\left(T \times B^{*}\right),
$$

where $\pi(S) \cap \pi\left(A^{*}\right)=\pi(T) \cap \pi\left(B^{*}\right)=\emptyset, S=\left[S, B^{*}\right]$, and $T=\left[T, A^{*}\right]$. In particular, if for some prime $p$ the subgroup $H$ contains a non-cyclic Sylow $p$-subgroup $P$, then $S$ and $T$ are $p^{\prime}$-subgroups of $G$.

Since $P=A_{p} B_{p}$ with $A_{p}=A \cap P$ and $B_{p}=B \cap P$, both subgroups $A_{p}$ and $B_{p}$ are non-trivial and so $p \notin \pi(S) \cup \pi(T)$. Therefore, if $G_{p}$ is a non-cyclic Sylow $p$-subgroup of $G, S_{p}=G_{p} \cap S$, and $T_{p}=G_{p} \cap T$, then up to conjugation $G_{p}$ coincides with one of the following subgroups of $G: P=A_{p} B_{p}, T_{p} \rtimes A_{p}$, $S_{p} \rtimes B_{p}$, and $S_{p} \rtimes T_{p}$. In particular, the Sylow $p$-subgroups of $G$ have rank at most 2 for $p>2$ (see [4, Satz III.11.5]) and at most 3 for $p=2$ (see [6, Theorem 5.1]). We now show that $G$ is in fact a group of rank at most 3 .

Indeed, suppose the contrary and let the group $G$ contain a subgroup $K$ whose minimal number of generators $d(K)$ is at least 4 . Since the Sylow subgroups of odd orders in $K$ have rank at most 2 by what was noted above, each Sylow 2-subgroup $Q$ of $K$ must have rank 3 by Lemma 2.7. It is clear 
that $Q=K \cap P$ for a Sylow 2-subgroup $P=A_{2} B_{2}$ of $G$. As the group $G$ is metabelian, the derived subgroup $P^{\prime}$ is abelian and normal in $G$. Moreover, $P^{\prime}$ has rank at most 2 by [6, Theorems 4.2 and $\left.4.3(\mathrm{e})\right]$.

Put $N=P^{\prime} \cap Q$. As $Q=K \cap P$, we have $N=K \cap P^{\prime}$ and so $N$ is an abelian normal subgroup of $K$ with rank at most 2 . In addition, $N \neq 1$, because otherwise the subgroup $Q$ is embedded in the factor group $P / P^{\prime}$ whose rank is equal to 2. On the other hand, since the factor group $Q / N=Q / P^{\prime} \cap Q$ is isomorphic to the factor group $Q P^{\prime} / P^{\prime} \leq P / P^{\prime}$, it is abelian of rank at most 2. Therefore, the Sylow 2-subgroups of the factor group $K / N$ have rank at most 2 and hence $K / N$ has rank at most 3 by Lemma 2.7. In particular, $d(K / N)<d(K)=4$ and so $N$ is not contained in the Frattini subgroup $\Phi(K)$ of $K$, because otherwise $d(K / N)=d(K)$. Clearly, passing to the factor group $K / \Phi(K)$, we may assume that $\Phi(K)=1$. Then the normal subgroup $N$ is complemented in $K$ and so in $Q$ by [4, Hilfsatz 3.4.4]. Moreover, since $\Phi(N)=1$ by [4, Hilfsatz 3.3.b], the subgroup $N$ is elementary abelian of order at most 4 .

Let $L$ be a complement to $N$ in $K$ and $M=Q \cap L$. Then $M$ is a Sylow 2-subgroup of $L$ and $Q=N \times M$, so that $M$ is abelian with $d(M) \leq 2$. Since the subgroup $K$ is supersoluble, its maximal subgroup $U$ of odd order is normal in $K$ and centralizes $N$. Therefore, $K=U \times Q=(U \times N) \rtimes M$ and hence the centralizer $C_{N}(M)$ is a non-trivial central subgroup of $K$. As $\Phi(K)=1$, the subgroup $C_{N}(M)$ is complemented in $K$ and thus in $Q$. From this, it follows that $Q=M \times N$ is abelian and $N$ is a central subgroup of $K$. Therefore, $K=(U \rtimes M) \times N$ and the subgroup $U \rtimes M$ is three-generated by Lemma 2.7. This means that there exist elements $u, v, w$ of $U$ and $x, y, z$ of $M$ such that $U \rtimes M=\langle u x, v y, w z\rangle$. Then $M$ modulo $U$ is generated by $x, y, z$. In particular, if $d(M)=1$, without loss of generality we may assume that $M=\langle x\rangle$ and $y=z=1$, so that $U \rtimes M=\langle u x, v, w\rangle$. In the case $d(M)=2$ we can take $M=\langle x, y\rangle$ and $z=1$. Then $U \rtimes M=\langle u x, v y, w\rangle$.

Finally, since $Q=M \times N$ is a 2-subgroup of rank 3 as noted above, only two cases are possible: either $M=\langle x\rangle$ and $N=\langle a, b\rangle$ has order 4 or $M=\langle x, y\rangle$ with $x \neq 1 \neq y$ and $N=\langle a\rangle$ is of order 2. Therefore, $K=\langle u x, a v, b w\rangle$ in the first case and $K=\langle u x, v y, a w\rangle$ in the second case. In both cases $d(K)<4$ and this contradiction completes the proof.

4. Products of a periodic and a torsion-free locally cyclic group. Recall that a group $G$ has finite torsion-free rank if it has a series of finite length whose factors are either periodic or infinite cyclic. The number $r_{0}(G)$ of infinite cyclic factors in such a series is an invariant of $G$ called its torsion-free rank. In this section, we describe the structure of the group $G=A B$ with locally cyclic subgroups $A$ and $B$, the first of which is periodic and the other non-trivial torsion-free. Clearly $r_{0}(B)=1$ and we note first that $r_{0}(G)=1$.

Lemma 4.1. Let $G=A B$ be a group with subgroups $A$ and $B$ such that $A$ is periodic abelian and $B$ is non-trivial torsion-free locally cyclic. Then $r_{0}(G)=$ 1. 
Proof. It was proved by Zaitsev [11, Theorem 3.7] (see also [1, Lemma 7.1.2]) that there exists a non-trivial normal subgroup of $G$ contained in $A$ or $B$. Therefore $G$ has the normal series $A_{0}<A_{0} B_{0}<G$ in which $A_{0}$ is the core of $A$ in $G$ and $B_{0}$ is the core of $B$ in $G$ modulo $A_{0}$. As is easily seen, the factors $A_{0}$ and $G / A_{0} B_{0}$ are periodic and the factor group $A_{0} B_{0} / A_{0}$ is isomorphic to $B_{0}$. Thus $r_{0}(G)=r_{0}(B)=1$, as claimed.

The following lemma is a consequence of the well-known theorem of I. Schur on the finiteness of the derived subgroup of a group that is finite over its center (see [8, Corollary to Theorem 4.12]).

Lemma 4.2. If a group $G$ contains a central subgroup $Z$ such that the factor group $G / Z$ is locally finite, then the derived subgroup of $G$ is locally finite.

Theorem 4.3. Let the group $G=A B$ be the product of two locally cyclic subgroups $A$ and $B$ such that $A$ is periodic and $B$ is non-trivial torsion-free. Then one of the following statements holds.

(1) The subgroup $A$ is normal in $G$ and so $G=A \rtimes B$;

(2) $A=A_{1}\langle a\rangle$ with $a^{2} \in A_{1}$, the subgroup $A_{1}$ is normal in $G$ and $G=$ $\left(A_{1} \rtimes B\right)\langle a\rangle$ with $b^{a}=b^{-1} \phi(b)$ for all $b \in B$, where $\phi: B \rightarrow A_{1}$ is a derivation of $B$ into $A_{1}$.

Proof. It is easy to see that each periodic normal subgroup $H$ of $G$ is contained in $A$, because $A H=A(A H \cap B)$ and $A H \cap B=1$. Therefore the core $A_{1}=$ $\cap_{g \in G} A^{g}$ of $A$ in $G$ is the maximal periodic normal subgroup of $G$.

Assume first that $A_{1}=1$ and let $B_{1}$ be the core of $B$ in $G$. Then $B_{1} \neq$ 1 by the theorem of Zaitsev noted above and so the factor group $G / B_{1}$ is periodic, because it is the product of two periodic subgroups $A B_{1} / B_{1}$ and $B / B_{1}$. Moreover, since the centralizer $C_{G}\left(B_{1}\right)$ of $B_{1}$ in $G$ contains $B$, the group $G$ induces on $B_{1}$ a periodic group of automorphisms which is isomorphic to the factor group $A / C_{A}\left(B_{1}\right)$. As is well-known, a periodic group of automorphisms of any locally cyclic torsion-free group is of order 2 . Therefore the order of $A / C_{A}\left(B_{1}\right)$ does not exceed 2 and hence either $A=C_{A}\left(B_{1}\right)$ or $A=C_{A}\left(B_{1}\right)\langle a\rangle$ with $a \in A$ and $a^{2} \in C_{A}\left(B_{1}\right)$.

On the other hand, since the centralizer $C_{G}\left(B_{1}\right)=C_{A}\left(B_{1}\right) B$ is normal in $G$ and periodic over $B_{1}$, its derived subgroup $C_{G}\left(B_{1}\right)^{\prime}$ is periodic by Lemma 4.2 and normal in $G$. Therefore $C_{G}\left(B_{1}\right)^{\prime} \leq A_{1}=1$ and hence $C_{G}\left(B_{1}\right)=C_{A}\left(B_{1}\right) \times$ $B$. But then again $C_{A}\left(B_{1}\right)$ is normal in $G$ and so $C_{A}\left(B_{1}\right)=1$. Thus in the case $A_{1}=1$ we have either $A=1$ and $G=B$ or $A=\langle a\rangle$ with $a^{2}=1$ and $G=B \rtimes\langle a\rangle$ with $b^{a}=b^{-1}$ for all $b \in B$.

Finally, returning now to the general case, we derive that either $G=A \rtimes B$ or $\left.G=\left(A_{1} \times B\right)\langle a\rangle\right)$ with $b^{a}=\phi(b) b^{-1}$ for every $b \in B$ and some element $\phi(b) \in A_{1}$. Moreover, since $\phi(b c)(b c)^{-1}=(b c)^{a}=b^{a} c^{a}=\left(\phi(b) b^{-1}\right)\left(\phi(c) c^{-1}\right)=$ $\left(\phi(b) \phi(c)^{b}\right)(b c)^{-1}$, it follows that $\phi(b c)=\phi(b) \phi(c)^{b}$ for any $b, c \in B$. The latter means in particular that the mapping $\phi: B \rightarrow A_{1}$ is a derivation of $B$ into $A_{1}$, as claimed.

5. Products of finitely many periodic locally cyclic groups. A well-known theorem of Huppert cited in the introduction says that every finite group of 
the form $G=A_{1} A_{2} \cdots A_{n}$ with pairwise permuting cyclic subgroups $A_{i}$ for $1 \leq i \leq n$ is supersoluble. This result was later extended to products of pairwise permutable locally cyclic Chernikov groups by Tomkinson [10]. He proved that in this case $G=A_{1} A_{2}, \ldots, A_{n}$ is a locally supersoluble Chernikov group. In this section, we generalize this result to products of arbitrary periodic locally cyclic groups. Recall that a group is said to be hyperabelian (respectively, hypercyclic) if it has an ascending series of normal subgroups with abelian (respectively cyclic) factors.

Lemma 5.1. Let $G=A_{1} A_{2} \cdots A_{n}$ be the product of pairwise permutable periodic locally cyclic subgroups $A_{i}$. If the set $\pi=\bigcup_{i=1}^{n} \pi\left(A_{i}\right)$ is finite, $p$ is the largest prime in $\pi, P_{i}$ is the Sylow p-subgroup of $A_{i}$, and $Q_{i}$ is the $p$ complement to $P_{i}$ in $A_{i}$ for each $1 \leq i \leq n$, then $G$ is a $\pi$-group, $P=$ $P_{1} P_{2} \cdots P_{n}$ is a normal Sylow p-subgroup of $G$, and $Q=Q_{1} Q_{2} \cdots Q_{n}$ is a p-complement to $P$ in $G$.

Proof. Since each of the $A_{i}$ is a subgroup of Prüfer rank 1, the group $G=$ $A_{1} A_{2} \cdots A_{n}$ is hyperabelian of finite Prüfer rank by [2, Theorem 3.1]. Therefore, arguing by induction on $n$ and applying [1, Corollary 3.2.7], and [2, Lemma 3.2], we derive that $G$ is a $\pi$-group, $P=P_{1} P_{2} \cdots P_{n}$ is a Sylow $p$-subgroup of $G$ and $Q=Q_{1} Q_{2} \cdots Q_{n}$ is a complement to $P$ in $G$. Moreover, taking into account that the subgroups $A_{i} A_{j}$ are locally supersoluble by Lemma 2.3 , we conclude that $P_{i}{ }^{A_{j}} \leq P$ for all $i, j$ and so $P$ is a normal subgroup of $G$.

Lemma 5.2. Let $G=A_{1} A_{2} \cdots A_{n}$ be the product of pairwise permutable locally cyclic subgroups $A_{i}$. If the group $G$ is periodic and the set $\pi(G)$ is finite, then $G$ is locally supersoluble.

Proof. Since $\pi(G)$ is finite, every locally cyclic subgroup $A_{i}$ is a Chernikov group, i.e. a finite extension of a direct product of finitely many quasicyclic subgroups. Therefore $G$ is a locally supersoluble Chernikov group by [10, Theorem B].

Proof of Theorem 1.3. Let $G=A_{1} A_{2} \cdots A_{n}$ be the product of pairwise permutable periodic locally cyclic subgroups $A_{i}$. Then $G$ is a periodic group by Lemma 5.1. If the set $\pi(G)$ is finite, then the group $G$ is locally supersoluble hypercyclic by Lemma 5.2. In the other case the set $\pi(G)$ is infinite and thus it can be presented as a union $\pi(G)=\bigcup_{i=1}^{\infty} \pi_{i}$ of finite subsets $\pi_{i}$ such that $\pi_{i} \subset \pi_{i+1}$ for all $i \geq 1$. Let $P_{i j}$ be the Sylow $\pi_{i}$-subgroup of $A_{j}$ for $1 \leq j \leq n$ and $G_{i}=P_{i 1} P_{i 2} \cdots P_{i n}$. Then $G_{i}$ is a Sylow $\pi_{i}$-subgroup of $G$ by Lemma 5.1 which is locally supersoluble as a group for each $i \geq 1$ by Lemma 5.2. Since $G=\bigcup_{i=1}^{\infty} G_{i}$, the group $G$ is also locally supersoluble, as claimed.

Acknowledgements. The second author likes to thank the Institute of Mathematics of the University of Mainz for its excellent hospitality during the preparation of this paper

Funding Open Access funding enabled and organized by Projekt DEAL. 
Open Access. This article is licensed under a Creative Commons Attribution 4.0 International License, which permits use, sharing, adaptation, distribution and reproduction in any medium or format, as long as you give appropriate credit to the original author(s) and the source, provide a link to the Creative Commons licence, and indicate if changes were made. The images or other third party material in this article are included in the article's Creative Commons licence, unless indicated otherwise in a credit line to the material. If material is not included in the article's Creative Commons licence and your intended use is not permitted by statutory regulation or exceeds the permitted use, you will need to obtain permission directly from the copyright holder. To view a copy of this licence, visit http://creativecommons. org/licenses/by/4.0/.

Publisher's Note Springer Nature remains neutral with regard to jurisdictional claims in published maps and institutional affiliations.

\section{References}

[1] Amberg, B., Franciosi, S., de Giovanni, F.: Products of Groups. Clarendon Press, Oxford (1992)

[2] Amberg, B., Sysak, Y.: Groups factorized by pairwise permutable abelian subgroups of finite rank. Adv. Group Theory Appl. 2, 13-24 (2016)

[3] Hartley, B.: Splitting over the locally nilpotent residual for a class of locally finite groups. Quart. J. Math. 27, 395-400 (1976)

[4] Huppert, B.: Endliche Gruppen I. Springer, Berlin (1967)

[5] Huppert, B.: Über das Produkt von paarweise vertauschbaren zyklischen Gruppen. Math. Z. 58, 243-264 (1953)

[6] Janko, Z.: Finite 2-groups with exactly one nonmetacyclic maximal subgroup. Israel J. Math. 166, 313-347 (2008)

[7] Kovacs, L.G.: On finite soluble groups. Math. Z. 103, 37-39 (1968)

[8] Robinson, D.J.S.: A Course in the Theory of Groups, 2nd edn. Springer, New York (1996)

[9] Sysak, Y.: Products of locally cyclic torsion-free groups. Algebra Logika 25, 672686 (1986)

[10] Tomkinson, M.J.: Products of abelian subgroups. Arch. Math. (Basel) 47, 107112 (1986)

[11] Zaitsev, D.I.: Products of abelian groups. Algebra Logika 19, 150-172 (1980)

Bernhard AmBERG

Institut für Mathematik

Johannes Gutenberg-Universität Mainz

55099 Mainz

Germany

e-mail: amberg@uni-mainz.de 
YAROSLAV SySAK

Institute of Mathematics

Ukrainian National Academy of Sciences

Kiev 01601

Ukraine

e-mail: sysak@imath.kiev.ua

Received: 11 September 2020

Revised: 16 January 2021

Accepted: 12 February 2021. 